\title{
Physical Layer Network Coding For Bridge Wireless Monitoring
}

\author{
A.M. Mejri, G.R. Rekaya \& J-C.B. Belfiore \\ Telecom-ParisTech, 46 Rue Barrault, 75013, Paris, France
}

\begin{abstract}
Structural Health Monitoring of bridges has aroused the interest of many researchers on various disciplines. Our purpose in this paper is to make an overview on SHM of bridges from two different research areas: Civil and Communication Engineering. For the former part, we provide some insights into the objectives and the ways SHM of bridges is made. For the Communication Engineering side, we provide some background on the used technologies in SHM of bridges. We distinguish the wired and Wireless Sensor Networks (WSNs). We focus on communication limitations in WSNs, mainly the interference problem occurring when the sensors broadcast their measurements and resulting in performance degradation. In this paper we review basic tools existing in literature based on the Physical Layer Network Coding approaches. We focus on the Compute-and-Forward protocol known to provide significant gains. Our main contribution concerns the implementation of this protocol and discussion of its practical challenges.
\end{abstract}

\section{INTRODUCTION}

Structural Health Monitoring (SHM) of bridges has drawn considerable interest of researchers in both civil and communication engineering fields. Several systems have been developed to ensure continuous bridge inspection in order to better understand the performance of the bridge, detect its failure and predict its remaining life time (Nigbor, 2000). Initial SHM tools were based on human visual inspection. Then, with the declining costs of electronics and emergence of computing technologies, sensor technologies changed fundamentally the way SHM was made. Wired Sensor Networks have been deployed to get real time measurements that describe the behavior of the bridge and allow to monitor its changing conditions such that the needed corrective actions can be made on time. The huge cables used to connect the different sensors drive their instrumentation costs very high and make their use limited. Recently, Wireless Sensor Networks (WSNs) technologies have emerged and impacted the field of Wireless monitoring. They have been used in several applications, like SHM. Wireless sensors gather the underlying physical measured properties and transmit the numerical data to a gateway through a multihop cooperative relay network. In such networks, two major problems are encountered. The first one is that the wireless sensor nodes, being energy-limited, have to consume as low as possible energy to maximize the whole network life time. The second one is that when data is wirelessly transmitted from one node to another, an interference problem may occur and considerably impacts the transmission rates and make the network suffer from a loss in spectral efficiency.

In this paper, we provide an overview on SHM from Civil Engineering and Communication Engineering points of view. The aim of the Civil Engineering part of the work is to outline the basic considerations concerning the relevance of SHM of bridges, the important parameters that have to be measured and the way the monitoring is performed. As far as the Communication Engineering field is concerned, we review the essential SHM systems based on sensor technologies, namely the wired and wireless sensor networks. We review their salient features, their use proposed so far in literature as well as their main shortcomings. We focus our interest on the WSNs technologies given their considerable advantages compared to the wired systems, and address the communication limitations due to the interference problem. Much of the research to date in wireless communications has focused on the so called Physical Layer Network Coding (PLNC) approach to struggle the interference. We provide 
through this work a review on the principle of PLNC and introduce an important PLNC protocol, called the Compute-and-Forward (CF). This protocol has recently drawn considerable interest of wireless communication researchers since it, theoretically, affords powerful resilience to interference and generates interesting gains in terms of network throughput. In this paper, we bridge theory with practice. We propose a real implementation of $\mathrm{CF}$ and discuss its practical aspects that have to be satisfied such that the promised powerful achievements of this protocol can be realized.

The flow of the remaining paper is organized as follows. In section 2 we point out the importance of SHM of bridges. In section 3 we outline the use of WSNs to perform bridge monitoring, their relevant advantages as well as their main drawbacks. Finally we exhibit the use of PLNC for interference mitigation and implementation issues related to the Compute-and-Forward in section 4.

\section{STRUCTURAL HEALTH MONITORING OF BRIDGES}

\subsection{Why to monitor?}

Aging, degradation, overloads and increasing train and vehicles' speeds confronted by transportation infrastructures in general, and bridges in particular, appeal for public safety concerns. Since the replacement of such structures is almost infeasible, and their maintenance and repair are extremely expensive, a salient call for Structural Health Monitoring is to date in order to ensure safety of passengers and vehicles (Dargie \& Poellabauer, 2010). SHM systems provide data from the inside of the bridge to allow a better understanding of its performance and a prediction of its durability and remaining life time (Nigbor, 2000).

Continuous SHM systems have the potential, by providing reliable information about the real behavior of the bridge, its condition and changes, to enable intelligent decision making, local and global damage detection at early stage. Thus immediate repair and corrective actions can be made before any collapse occurs and allow avoiding considerable risks of further damage.

The importance of SHM systems can also be attributed to their ability to provide effective measurements of the response of the bridge to external environmental impacts. Indeed, the service life of a bridge is seriously shortened with environmental effects and events like floods, earthquakes, overload (Lynch, 2004). Thus, an important point that must not be overlooked is to perceive in details the response of the bridge to such impacts and monitor what's happening in real time such that the bridge owners are enabled to analyze study and estimate the time-scale and severity of any changes occurring on the bridge through its life time.

\subsection{What to monitor?}

SHM systems applied to bridges are in essence used to collect data characterizing the real time behavior of the bridge (Lynch, 2004). The monitoring process can be related to: i) monitoring the space around the bridge, ii) monitoring the physical properties of the bridge, iii) monitoring the interaction between them i.e. the impact of the external events on the bridge. Monitoring wind power, temperature, humidity and air pollution belongs to the first category. Monitoring the operating vibration of the bridge (Kijewski, Su, Abittan \& Antsaklis, 2006), the fatigue level damage (Bischoff, Meyer, Enochsson, Feltrin \& Elfgren, 2009), the concrete strain, the joint displacement cracking and moisture are examples of monitoring physical properties of the bridge. And enormous data about the impact of the environmental surroundings on the bridge can be collected and estimated like measuring earthquake ground motion and shaking, and estimating the wind-induced vibration. Detection and localization of such hazards allow performing an up-to-date inspection of the bridge and alarming the monitoring professionals when the damage level overcomes some threshold.

\subsection{Where to monitor?}

In order to meet the objectives of SHM of bridges, designed systems need to be installed in appropriate and strategic positions adapted to the environment, very close or inside the bridge to monitor in order to be able to detect both local and global properties and inspect the area of interest with fine granularity and high level accuracy. An important issue of the system life time must not be overlooked especially when sensor technologies are deployed inside the bridge to perform SHM.

\subsection{When to monitor?}

Bridge monitoring and data collection can be timetriggered or event-triggered (Dargie \& Poellabauer, 2010). For the first case, data collection takes place periodically at regular intervals. The parameters of interest, like temperature or strain, are not subject to rapid or sudden changes, and their periodic collection is enough. If any abnormalities are detected, an alarm is generated to alert the inspectors about the occurring events. In contrast, several parameters may change suddenly and speedily. Their eventtriggered collection is of a great importance since it can signal a noteworthy safety hazard. Detection of seismic activities is an example of such parameters (Nigbor, 2000). 


\subsection{How to monitor?}

Visual inspections were widely used to inspect outward signs of deterioration of a bridge. However, these techniques suffer from many drawbacks among which we refer two principle ones. First, these techniques are labor intensive tasks requiring an onsite evaluator. Second, since the assessment depends on the level of experience of engineers involved in the inspection process, it loses its subjectivity.

Alternative SHM systems are based on Nondestructive Evaluation (NDE) technologies including Acoustic Emission (AE), Ultrasonic Testing and Radar Tomography. Although these techniques seemingly provide significant means for bridge inspection, an important point that must not be missed is that they require the knowledge of damage locations which calls for additional tasks (Grosse \& Krger, 2008).

Alternatively, wired permanent SHM systems are used. The core principle of these systems is to use wired sensor technologies (Lynch, 2004). A variety of sensors are used to measure physical properties of interest, and they are connected through extensive lengths of cables for measurement transfer to a central base station to make necessary analysis, storage or processing on the collected data.

In spite of their reliability to provide efficient bridge inspection, the wired SHM systems suffer from several shortcomings. First, their dependence on the extensive lengths of wires used to interconnect the different components of the system drives their installation, maintenance and cable protection costs very high compared to the cost of the bridge. In addition, these systems are vulnerable to damage, time consuming, difficult to install and high power consuming. Furthermore, in wired sensors based SHM, removing and addition of sensors in an existing network is an inconvenient task and a changing topology due to the increase of the number of sensors generate many practical difficulties and poses several challenges to the design and management of the wired sensor network.

Wireless Sensor Networks represent a viable substitute for traditional wire-based SHM systems and provide elegant solutions to problems that were traditionally considered tough. Indeed, a large number of tiny, cheap wireless sensors can be easily deployed to sense and collect enormous data about parameters of interest with fine granularity. The wireless sensors can be placed on, under or within the bridge, in locations which may be extremely difficult to access. Their low cost, alleviated instrumentation and maintenance costs, design flexibility and reliability make them have the potential to fundamentally change the way SHM of bridges is made.

In the focus of this work, we give deeper insights into the use of WSNs Technologies for bridge moni- toring. We provide within the next section an overview on their promising advantages, discuss their drawbacks and present a communication scheme that affords means to handle and ameliorate their shortcomings.

\section{BRIDGE MONITORING USING WIRELESS SENSOR NETWORKS}

\subsection{WSNs technologies}

A WSN consists in several nodes (tens to thousands) called motes. They have on board one or several sensors automatically interconnected through wireless channels. They communicate with each other and with a base station called sink node responsible of collecting the sensors' measurements and forwarding them to remote centers, via a gateway or via internet connections, for further processing, storage and analysis. We provide through Figure 1 an illustration of a Wireless Sensor Network.

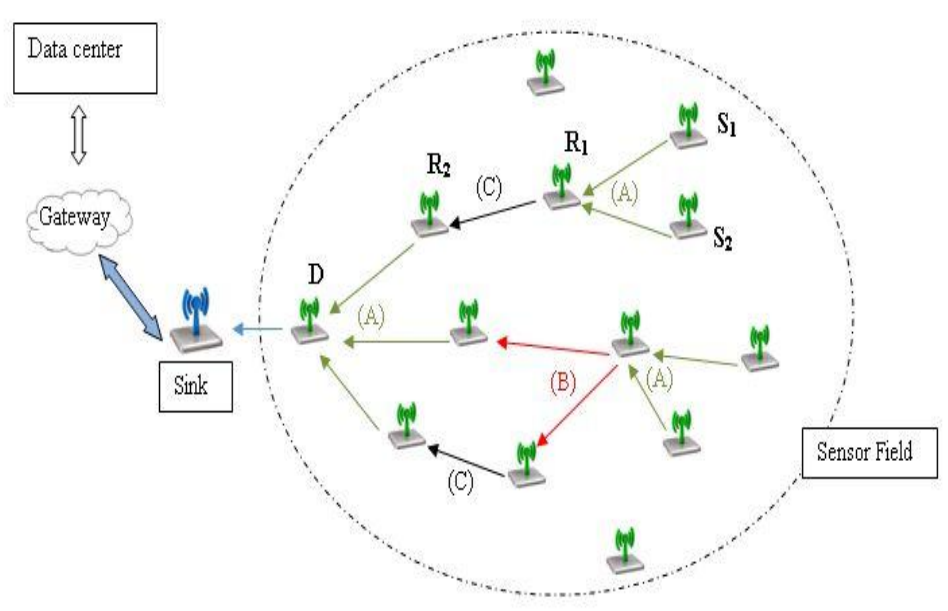

Figure 1. Wireless Sensor Network.

Several types of sensors have been developed for bridge monitoring applications, like accelerometer, strain gages, displacement sensors, tilt sensors, wind speed and direction sensors as well as temperature sensors (Lynch, 2004). The wide use of these technologies and their fast adoption is mainly attributed to their salient features. Indeed, in contrast to traditionally used wired SHM systems, the WSNs are low cost, easy and fast to deploy, flexible, robust, autonomous, self-configuration and energy harvesting systems. Moreover, the ability to massively deploy a large number of sensors for bridge monitoring affords a high sensing quality, thus a fine grain of monitoring. In addition, the wireless platform of the network allows a remote administration; calibration and reprogramming of the sensor nodes in order to keep the whole system flexible, up-to-date and easy to manage. 


\subsection{WSNs for bridge monitoring}

\subsubsection{Related works}

An overview on the existing wireless monitoring systems based on WSNs was driven in (Lynch, 2004). Authors in (Whelan, Gangone, Janoyan, Cross \& Jha, 2007) and (Whelan, Fuchs, Gangone \& Janoyan, 2007) have been interested in the strain response and dynamic properties of bridge structures. They provide a Wireless System Solution based on WSNs. This solution is developed in Clarkson University and validated by the means of dense field deployments and laboratory testing. Inspection of the growth of cracks in bridges has been the focus of (Kotowski, Dowding \& Fuller, 2009), in which authors propose a WSN-based design to continuously and automatically measure the elongation of cracks. The reliability of this system has been tested and has been proved its ability to afford remote real-time monitoring and alerting about significant crack growths. WSNs have been also used for bridge crack localization (Zhong \& He, 2008).

Inspection and detection of the bridge status and behavior are performed through the interpretation of the vibration and strong motion measurements collected using a WSN system (Sukun and al, 2007). The reliability of the proposed system is successfully validated through real tests on the Golden Gate Bridge in San Francisco. Further interesting works carried out in (Hakman et al, 2010) focus on damage detection and localization. The proposed approach is based on a low power consumption co-design integrating WSN platform and structural algorithms. Real experiments are carried out on a physical Steel beam at Washington University of Structural control and Earthquake Engineering to evaluate the design's efficiency in multilevel damage localization. In (Bischoff, Meyer, Enochsson, Feltrin \& Elfgren, 2009), WSNs are used for fatigue assessment in Steel railway bridges. Works of (Nigbor, 2000) were dedicated to investigate instrumentation tools including wireless sensor technologies for diagnostics of seismic response of bridges. Another issues related to dynamic monitoring of bridges were independently addressed in (Nigbor, 2000) and (Kalayni \& Chellappan). As far as the power consumption problem is concerned, several works have been proposed in literature including a low power consumption platform using smart brick WSNs (Harms, Segidh \& Bastianini, 2010), and installation of solar power WSN on the Gold star Bridge in New London and in Ben Franklin Bridge (Williston \& Vermont, 2007).

\subsubsection{WSN communication design}

In a WSN, sensor nodes are the data sources; they gather the measured parameters and transmit the numerical data to the sink node using wireless links. Each node has its coverage radio wave. Nevertheless, due to the limitations of the communication range, sensor nodes find a way for better quality of transmission and take advantage of wireless communications to forward data in a Mulithop fashion, i.e. data from a node can jump through multiple nodes to be eventually delivered to the sink node. For example, as shown in Figure 1, data from the sensor node $S_{1}$ is relayed by $R_{1}$ and $R_{2}$ to reach the destination node $\mathrm{D}$, and then it is delivered to the sink node. WSNs support Multihop Relaying because it allows avoiding long distances which are expensive in terms of power consumption and complexity of implementation, struggle against propagation and fading effects, and therefore keeps the life time of the network maximized. Meanwhile, in order to take advantage of the powerful gains and tools provided by the WSNs technologies for wireless monitoring, several challenges have to be met and many requirements have to be satisfied.

\subsubsection{System constraints and requirements}

We can divide the major pertaining constraints into two categories. The first one concerns the design constraints, and the second is related to the communication limitations. The key design requirement is the power efficiency. Indeed, the sensor life time shows a strong dependence to the battery life time. The different tasks performed by the sensor's components need to be efficiently conceived to keep the network life time maximized. In particular, the wireless radio unit, the largest power consumer among all the components, needs to be optimally designed and used. Awareness refers also to the fault tolerance and scalability. In other words, the tasks of the whole WSN should not be affected by the failure, addition or removing of some sensors.

Now, as far as the communication limitations are concerned, first relevant constraints that inherently limit the wireless communications are the low available bandwidth and transmission rates. Moreover, from a communication channels point of view, real wireless systems in general, and WSNs in particular, need to solve a plethora of practical aspects related to interference issues. Indeed, considering a Multihop relaying system, two phenomena occur and lead to interference and data loss. They are basically the broadcast and superposition properties of the wireless medium. The former makes both desired receiver node and other nodes within the same transmission range receive the transmitted data. At the non desired receiver node, superposition of both desired and non desired information messages requires additional processing such that the useful data can be adequately decoded. As illustrated in Figure 2, the destination node $D_{1}$ is interested only in receiving the message $x_{1}$ sent by its corresponding source $S_{1}$. However, the broadcast of $x_{2}$ by the source $S_{2}$ makes $\mathrm{D}_{1}$ receive both $\mathrm{x}_{1}$ and $\mathrm{x}_{2}$ (a combination of them) and unable to distinguish its desired data. This interference issue makes the whole network suffer from a 
loss in spectral efficiency and unable to meet its promised potential.

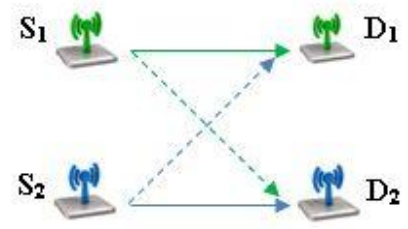

Figure2. Broadcast and superposition properties of the wireless medium.

Interference management in wireless networks has recently drawn considerable interest of the communication research community through the development of the so called Wireless Network Coding (Kuo, 2009),(Wu, 2007). This approach has the potential to radically change the way the interference management is performed. In this paper, we are interested in the type of communication that allows efficient, interference-free and reliable data transfer between nodes in a WSN.

\section{NETWORK CODING FOR BRIDGE MONITORING}

\subsection{Problem setup}

Once the network infrastructure is created, wireless communication links are established to enable the sensor nodes share and transfer their data. Different types of channels characterize the internodes wireless communications. As illustrated in Figure 1, they are mainly three channels:

- Multiple Access Channel (channel (A)): in this channel, two or multiple sources send their information to the same receiver node (relay $\mathrm{R}$ in the figure). - Broadcast channel (channel (B)): in this channel, source information is broadcasted to all the nodes within the coverage range.

- Point-to-point channel (channel (C)): it characterizes the transmission of the information message from one node to another one.

In this work, we focus our interest on the Multiple Access Channel (MAC) since it poses many challenges and requires solving a plethora of practical problems that impact the performance and the life time of the whole network (Mergen \& Tong, 2006). We consider a group of $\mathrm{N}$ sensors $S_{\mathrm{i}}, \mathrm{i}=1, \ldots$, $\mathrm{N}$. Each sensor observes conditionally, independent and identically distributed (i.i.d) data $\mathrm{w}_{1}, \ldots, \mathrm{w}_{\mathrm{N}}$. Each sensor $S_{i}$ transmits a signal $\mathrm{x}_{\mathrm{i}}$ depending on its acquired observation using an encoding scheme. The transmitted signals are subjects to channel attenuations translated by the real (or complex) coefficients $h_{i}, i=1, \ldots, N . h_{i}$ are i.i.d zero-mean, unit-variance random Gaussian variables. Signals are received through a Gaussian Multi-access channel and the channel output, which corresponds to the received signal at the relay node, can be modeled as

$y=\sum_{i=1}^{N} h_{i} x_{i}+z$

where $\mathrm{z}$ denotes the additional Gaussian noise. The relay node, hence, receives a noisy combination of the original signals and attempts to reliably and efficiently decode them, or at least decode meaningful information (function) of them to be forwarded to following hops or eventually to the sink node if the relay is directly connected to the sink.

The communication problem we address in this paper is: How to make the relay node communicate the sensor measurements $x_{i}, i=1, . ., N$ such that the decoding process at the relay node satisfies the avoidness of the massive use of scarce resource like bandwidth and power?

In order to meet these targets, smart data processing at the relay is required. From this raises the interest of Network Coding (NC), a routing approach proposed initially for the multicast problem in wired computing networks. This approach has been used in literature for wireless sensor network applications (Guo, Xie, Cui \& Wang, 2006) to solve routing problems (Lopez Toledo \& Wang, 2007), (Yang, Zhong, Sun \& Yang, 2008), the exchange of information data inside a cluster of sensors (Xiong, Liu, Huang, Cheng \& Cheng, 2007) and for performing data collection (Wang, Zhang \& Liu, 2006). In the next subsection we review the principle of this concept and provide deeper insights about its ability to solve the problem of interference and its impacts on the performance of the WSN in interest.

\subsection{Network Coding: generalities}

Network Coding (NC) was first proposed for wired networks (Fragouli, Boudec \& Widmer, 2005), (Yeung et al., 2005). The aim is to enhance the channel capacity. Intermediate nodes in a multihop cooperative network are allowed to mix the received packets into one or several outgoing packets and send them to next hops or to destination nodes. NC can be considered as a new technique of performing routing, which allows achieving higher data rates. For the case of Linear Network Coding (LNC), output packets are linear combinations or functions of the received packets. LNC offers a linear framework that facilitates the coding and decoding algorithms and reduce the power consumption dedicated to processing tasks.

In order to better explain the core principle of the $\mathrm{NC}$, we consider the example of the Wireless Exchange channel, where two end nodes $\mathrm{N}_{1}$ and $\mathrm{N}_{2}$ exchange information with the help of a relay node $\mathrm{R}$ since they don't belong to the region of possible transmissions of each other, and hence, they don't have direct link between them. In Figure 3 we illustrate the traditional Store-and-Forward scheme. In 
this scheme, the role of the relay sensor node is to store a copy of the received packet and forward it during the next time slot to the destination nodes. This scheme requires 4 time slots to make both destinations $\mathrm{N}_{1}$ and $\mathrm{N}_{2}$ receive the desired packets $\mathrm{p}_{2}$ and $\mathrm{p}_{1}$ respectively.

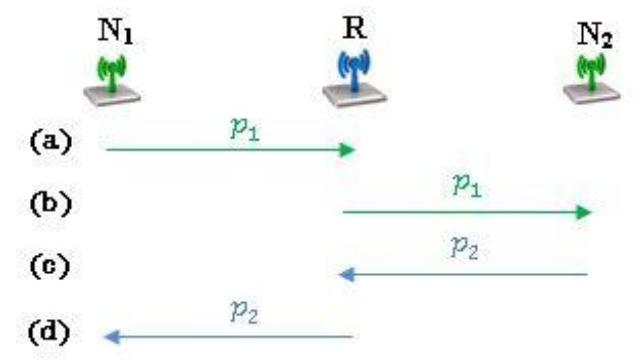

Figure 3. Store and Forward.

Consider now the communication scheme illustrated in Figure 4 in which Network Coding is applied on the Network Layer of the protocol stack, i.e. NC is applied on the information bits. The end nodes $\mathrm{N}_{1}$ and $\mathrm{N}_{2}$ send their packets $\mathrm{p}_{\mathrm{i}}, \mathrm{i}=1,2$ sequentially. The novelty now with the NC is that the relay node R, decodes each packet separately, i.e. $\mathrm{p}_{1}$ is decoded at time slot (a) and $\mathrm{p}_{2}$ during time slot (b). During the third time slot (c), R broadcasts the XOR mixture of the decoded bits, $\left(\mathrm{p}_{1}\right.$ XOR $\left.\mathrm{p}_{2}\right)$ to the end nodes. $\mathrm{N}_{1}$ and $\mathrm{N}_{2}$, having the side information, decode the desired information from the XOR combination. For example, $\mathrm{N}_{1}$ already knows the content of $\mathrm{p}_{1}$, while it receives $\left(\mathrm{p}_{1}\right.$ XOR $\left.\mathrm{p}_{2}\right)$, it performs $\mathrm{p}_{1}$ XOR $\left(\mathrm{p}_{1}\right.$ $\left.\mathrm{XOR} \mathrm{p}_{2}\right)=\mathrm{p}_{2}$ to get the wanted packet $\mathrm{p}_{2}$. The NCbased scheme in Figure 4 requires only three time slots to perform the information exchange, thus NC leads to the reduction of the transmission time and enhancement of the channel capacity. The XOR function can be generalized to any function. Among the interesting functions of the data measurements in WSNs applications, the mean, mode, media, max and min functions were studied in (Giridhar \& $\mathrm{Ku}$ mar, 2005).

(a)
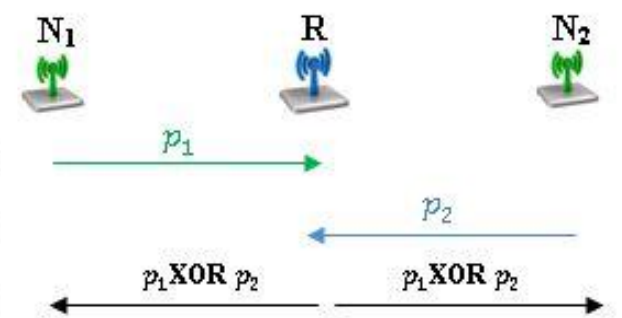

(b)

(c)

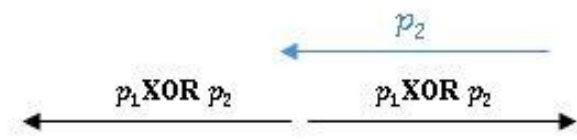

Figure 4. Network Coding in the Exchange Channel.

For both previous schemes, interference was avoided by transmission scheduling making the source sensor nodes transmit sequentially. However, in real wireless networks, due to the broadcast property of the wireless medium, transmitted signals are simultaneously received at the destination. Interference occurs and results in loss in performance and requires compensation and enhancement. This can be solved by the means of Physical Layer Network Coding. It represents the physical layer version of the Wireless Network Coding approach applied at the signal space in contrast to the Wireless Network Coding performed on the information bits.

PLNC turns the broadcast and superposition properties into boosting characteristics for achieving higher transmission rates and increasing the network throughput. To explain the PLNC, we take again the example of the Wireless Exchange Channel. In Figure 5 we illustrate the application of the PLNC.

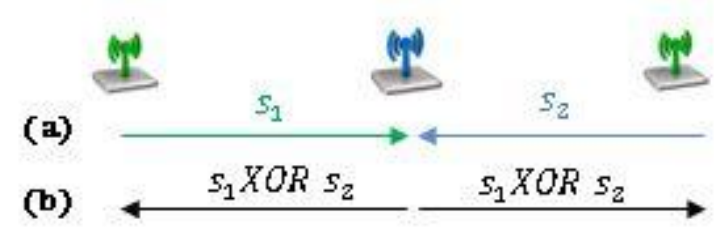

5. PLNC in the Exchange Channel.

Figure

The first difference with the previous schemes is that end nodes transmit their signals simultaneously, and the second difference is that the relay node doesn't decode the received signals separately; it rather decodes a function of them and broadcasts it to end nodes. The XOR function illustrated in Figure 5 can be generalized. PLNC exploits thus the interference provided by the channel to improve the spectral efficiency and reduce energy consumption for reliable and efficient transmissions. Different PLNC schemes have been proposed in literature to provide tools to mix the incoming combinations of original signals at intermediate nodes. We distinguish two major schemes: Modulation-demodulation based schemes (Hu \& Ibnkahla, 2010) and Channel Coding based schemes (Ke \& Jing, 2010).

In this work, we restrict our focus on a channel coding based PLNC protocol, named the Compute-andForward based on channel codes as salient tools to ensure reliable transmissions in presence of noise in wireless communications.

\subsection{Channel-coding based PLNC: the compute- and-forward protocol}

The Compute-and-Forward protocol has recently drawn considerable interest of researchers on wireless communication field (Nazer, 2009). It is a PLNC protocol in which a particular function of the original signals is computed. The core principle of this approach is based on harnessing the interference 
provided by the MAC channel in order to compute an integer, linear and noiseless combination of $\mathrm{x}_{\mathrm{i}}$, $i=1, . ., N$ (Nazer \& Gastpar, 2008). As a channel coding based scheme, an end-to-end channel coding is performed. The source nodes encode their data using lattice codes (Nazer \& Gastpar, 2007). The relevant characteristics that suggest the use of these codes are their good statistical and algebraic structures that allow approaching the capacity of point-to-point additive white Gaussian channels. After the encoding step, the constructed codewords cross the channel. The relay receives the noisy combination given in equation (1) and attempts to decode from y an integer, noiseless, linear combination in the form

$\lambda=\sum_{i=1}^{W} a_{i} x_{i}$

where $a_{i}, \mathrm{i}=1, \ldots, \mathrm{N}$ are the integer coefficients of the decoded function. This meaningful function provides resilience to interference and noise, which are the most obvious obstacles in wireless communications.

In order to get the function $\lambda$, three steps need to be performed:

- Step 1: Scaling of the channel output: The aim of this step is to make the channel output as close as possible to an integer linear combination of the original codewords $\mathrm{x}_{\mathrm{i}}$. Indeed, the adopted lattice encoding scheme outputs vectors $x_{i}$ and guarantees that any integer linear combination of these codewords is also a codeword. However, in our model, the linear combination of the codewords is no longer integer since $h_{i}$ are reals. By scaling the received signal y by a convenient factor $\alpha$, we get $\tilde{y}$ such that

$$
\begin{aligned}
& \tilde{y}=\alpha y=\alpha\left(\sum_{i=1}^{N} h_{i} x_{i}+z\right) \\
& =\underbrace{\sum_{i=1}^{N} a_{i} x_{i}}_{i=1}+\underbrace{\sum_{i=1}^{N}\left(\alpha h_{i}-a_{i}\right) x_{i}+\alpha z}_{\text {Effective Noise }}
\end{aligned}
$$

The choice of $\alpha$ brings the Effective noise very small.

- Step 2: Quantization step: choice of the function's coefficients: The desired integer function to decode has coefficients $a_{i}, i=1, \ldots, N$. Prior to decoding, a quantization step is necessary to fix these coefficients. In their work (Nazer \& Gastpar, 2008), Nazer and Gastpar propose a quantization method that guarantees a maximization of the Rate necessary to compute the function $\lambda . \mathrm{a}_{\mathrm{i}}$ are taken such that the computation rate is maximized.

- Step 3: Decoding of lattice Equation $\lambda$ : Once the coefficients $a_{i}$ are fixed, the remaining task consists in decoding $\lambda$ from the scaled signal $\tilde{y} . \lambda$ is also called lattice equation since it's a linear integer combination of lattice codewords. So $\lambda$ belongs to some lattice $\Lambda$. Now for the decoding, optimal approach based on the Maximum Likelihood (ML) Criterion can be used. It allows getting an optimal estimate of $\lambda$ that satisfies $\hat{\lambda}=\operatorname{argmax}_{\hat{\lambda \in \Lambda}} \mathrm{p}(\tilde{y} / \lambda)$

In practical settings, algebraic tools are needed to perform the ML decoding.

Theoretical breakthroughs of the Compute-andForward protocol seemingly answer to the problem of interference mitigation. However, to achieve this promised potential, a plethora of practical aspects need to be solved. Recent works on the CF have been only interested in the theoretical limits of this protocol. A main contribution of this work is to discuss implementation issues related to the CF. First issue to stress is related to the quantization step. Indeed, in our initial implementation of CF achieved in (Mejri, Rekaya \& Belfiore, 2012) we derived a strong dependence between the practical performance of the CF protocol and the quantization approach used to choose the function's coefficients. More reliable quantization techniques need to be investigated for performance enhancement. Now, the implementation of CF in real WSNs systems poses additional practical challenges related to the decoding techniques used in step 3. Indeed, the ML criterion-based decoder seems to be hard to implement. It arouses in practice several problems related to the lattice dimension, the choice of the lattice encoders to code the data measurements, as well as the decoding metric. These parameters impact the performance of the $\mathrm{CF}$ and make the use of suboptimal decoders a necessary alternative in practical settings. We are currently working on these research issues, specially we investigate efficient decoding techniques, quantization methods and coding schemes that enable real wireless systems like WSNs take advantage of the promised powerful gains provided by the $\mathrm{CF}$ for interference mitigation.

\section{CONCLUSION AND PERSPECTIVES}

In this paper, SHM of bridges was discussed based on two different expertise domains: Civil Engineering and Communication Engineering. Experts in the civil engineering field are interested in making a real-time inspection of the bridge. They try to optimize the structural elements to monitor, the place, the time and the tools of the monitoring process such that reliable and sustainable health inspection of the bridge can be achieved. In order to reach these targets, an interdisciplinary collaboration with Electrical and communication engineering researchers is a basic requirement. Indeed, the most used SHM systems so far deploy sensor technologies. Traditional systems have witnessed the use of wired sensor technologies and recently, WSNs have emerged and changed the way SHM was performed. In such systems, wireless sensors detect properties of interest and exploit the multihop topology of WSNs to forward data to remote centers. In this work, we made an overview on these SHM systems; we addressed 
the problem of interference existing in WSNs during the data dissemination. We introduced existing techniques in literature to solve this problem using the Network Coding concept. We briefly reviewed the principle of the compute-and-forward protocol. An essential contribution of this work concerns its practical implementation and related challenges that are necessary to solve in order to make its benefits in terms of interference mitigation in real WSNs systems achievable.

\section{ACKNOWLEDGMENTS}

This work was supported by the project SmartEN which has received funding from the European Union People Program (FP7/2007-2013), under grant agreement $\mathrm{n}^{\circ} 238726$.

\section{REFERENCES}

Bischoff, R., Meyer, J., Enochsson, O., Feltrin, G. \& Elfgren, L. 2009. Event-based Strain monitoring on a railway bridge with a Wireless Sensor Network. Fourth International Conference on Structural Health Monitoring of Intelligent Infrastructure, Switzerland 22-24 July 2009.

Dargie, W. \& Poellabauer, C. 2010. Fundamentals of Wireless Sensor Networks: Theory and Practice. Chichester: Wiley-Blackwell.

Fragouli, C., Boudec, J.L, \& Widmer, J. 2005. Network coding: an instant primer. ACM SIGCOMM Computer Communication Review 36(1): 63-68.

Grosse, C.U \& Krûger, M. 2008. Bridge Monitoring Using Wireless Sensors and Acoustic Emission Techniques. Proceedings, Inaugural International Conference of the ASCE Engineering Mechanics Institute.

Giridhar, A. \& Kumar, P.R. 2005. Computing and Com municating Functions Over Sensor Networks. IEEE Journal On Selected Areas in Communications.

Guo, Z., Xie, P., Hong Cui, J \& Wang, B. 2006. On Applying Network Coding to Underwater Sensor Networks. WUWNet.

Hackmann, G., Guo, W., Yan, G., Lu, C. \& Dyke, S. 2010. Cyber-Physical Codesign of Distributed Structural Health Monitoring With Wireless Sensor Networks. ICCPS.

Harms, T., Segidh, S. \& Bastianini, F. 2010. Structural Health Monitoring of Bridges Using Wireless Sensor Networks. IEEE Instrumentation and Measurement Magazine: 14-18.

Hu, P. \& Ibnkahla, M. 2010. A Survey Of Physical Layer Network Coding in Wireless Networks. Biennial Symposium on Communications: 311-314.

Kalayni, P. \& Chellappan, C. 2011. Heterogeneous Wireless Mobile Sensor Network Model Based Routing Adopted to Dynamic Topology. European Journal of Scientific Research: 143-150.

Ke, G. \& Jing, W. 2010. Improvement of Physical-Layer Network Coding Method in Relay Networks. International Conference On Communications and Mobile Computing: 457-460.

Kijewski-Correa, T., Su, S., Abittan, E., \& Antsaklis, p.J. 2006. On The Use Of Heterogeneous Wireless Sensor Nnetworks For Damage Assessment In Bridges Under Unkown Excitations. 4WCSCM: 11-13.

Kuo, F. C. 2009. Exploiting Network Coding in Lossy Wireless Networks. Thesis dissertation.
Lopez, A. \& Wang, X. 2007. Efficient Multipath in Sensor Networks using Diffusion and Network Coding. 40 ${ }^{\text {th }} \mathrm{An}$ nual Conference on Information Sciences and Systems: 8792.

Lynch, J. P. 2004. Overview of Wireless Sensors for RealTime Health Monitoring of Civil Structures. Proceedings of the $4^{\text {th }}$ International Workshop on Structural Control and Monitoring.

Mejri, A., Rekaya-Ben Othman, G. \& Belfiore, J-C. 2012. Lattice Decoding For The Compute-and-Forward Protocol, IEEE Third International Conference on Communications and Networking.

Mergen, G. \& Tong, L. 2006. Type Based Estimation over Multiaccess Channels. IEEE Transactions on Signal Processing: 613-626.

Nazer, B. \& Gastpar, M. 2007. The Case for Structured Random Codes in Network Communication Theorems. ITW.

Nazer B. \& Gastpar, M. 2008. Compute-and-Forward: Harnessing Interference Through Structured Codes. ISIT: 772776.

Nazer, B. 2009. Exploiting Interference Through Algebraic Strcuture. Thesis dissertation.

Nigbor, R.L. 2000. Instrumentation Systems for Dynamic Monitoring of Bridges. Invited Workshop on Instrumental Diagnostics of Seismic Response of Bridges and Dams.

Sukun, K., Pakzad, S., Culler, D., Demmel, J., Fenves, G., Glaser, S. \& Turon, M. 2007. Health Monitoring of Civil Infrastructures Using Wireless Sensor Networks. IPSN: 254-263.

Wang, D., Zhang, Q., \& Liu, J. 2006. Partial Network Coding: Theory and Application for Continuous Sensor Data Collection. IWQoS: 93-101.

Whelan, M. J., Fuchs, M. P, Gangone, M. V. \& Janoyan, K. D. 2007. Development Of a Wireless Bridge Monitoring System For Condition Assessment Using Hybrid Techniques. SPIE Smart Structures Symposium.

Whelan, M. J., Gangone, M.V., Janoyan, K.D. , Cross, K. \& Jha, R. 2007. Reliable High-Rate Bridge Monitoring using Dense Wireless Sensor Arrays. International Workshop on Structural Health Monitoring.

Williston, Vermont. 2007. Solar Powered Wireless Sensor Networks Monitor Bridge Spans. Williston, Vermont: Micro Strain Incorporation.

Wu, Y. 2007. Network Coding For Wireless Networks. Microsoft Research.

Xiong, Z., Liu, W., Huang, J., Cheng, W., Cheng, B. 2007. Network Coding Approach for Intra-cluster Information Exchange in Sensor Networks. $66^{\text {th }}$ IEEE Vehicular Technology Conference: 164-168.

Yang, Y., Zhong, C., Sun, Y. \& Yang, J. 2008. Energy Efficient Reliable Multi-path Routing Using Network Coding for Sensor Networks. IJCSNS 8(12).

Yeung R. W, Li, S., Cai, N. \& Zhang, Z. 2005. Network Coding Theory. Now Publishers.

Zhong, Z. \& He, T. 2008. Bridge Crack Localization Using Wireless Sensor Networks. In Inaugural International Conference of the Engineering Mechanics Institute. 